\title{
Faire voile et jeter l'ancre en Polynésie française
}

Quand les mobilités des plaisanciers au long cours interrogent les modes de circuler et d'habiter

\section{Nathalie Bernardie Tahir et Sarah Bernard}

\section{(2) OpenEdition}

\section{Journals}

Édition électronique

URL : https://journals.openedition.org/cdg/6037

DOI : $10.4000 /$ cdg. 6037

ISSN : 2107-7266

Éditeur

UMR 245 - CESSMA

Référence électronique

Nathalie Bernardie Tahir et Sarah Bernard, «Faire voile et jeter l'ancre en Polynésie française », Carnets de géographes [En ligne], 14 | 2020, mis en ligne le 01 janvier 2020, consulté le 22 mai 2021. URL : http://journals.openedition.org/cdg/6037 ; DOI : https://doi.org/10.4000/cdg.6037

Ce document a été généré automatiquement le 22 mai 2021

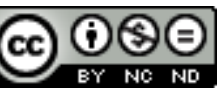

La revue Carnets de géographes est mise à disposition selon les termes de la Licence Creative Commons Attribution - Pas d'Utilisation Commerciale - Pas de Modification 4.0 International. 


\title{
Faire voile et jeter l'ancre en Polynésie française
}

Quand les mobilités des plaisanciers au long cours interrogent les modes de circuler et d'habiter

\author{
Nathalie Bernardie Tahir et Sarah Bernard
}

\section{Introduction}

« Le bateau, c'est la liberté, pas seulement le moyen d'atteindre un but, comme je le croyais il n'y pas si longtemps. Petite maison spartiate que j'emmène avec moi et qui m'emporte où je veux dans le monde, Marie-Thérèse représente maintenant la riche solitude des grands espaces où le passé et le futur se confondent pour devenir l'instant présent dans le chant de la mer » (Moitessier, p. 170).

1 Moitessier n'est pas qu'un écrivain rédigeant ses Mémoires au crépuscule de sa vie. Il a véritablement représenté un modèle pour plusieurs générations de "voileux » qui ont décidé de suivre les traces de ce navigateur exceptionnel, « vagabond des mers du sud » comme il aimait à se décrire, ayant fait deux fois le tour du monde avant de jeter l'ancre en Polynésie. Ce parcours, tel qu'il est raconté dans les nombreux ouvrages qu'il a rédigés, ne reflète pas seulement un itinéraire maritime, il est bien plus que cela : une aventure certes, un exploit sportif, incontestablement, mais surtout la quête d'une autre vie, d'une relation différente à l'autre, à soi, à la nature, à la société et au monde.

Dans le sillage de Moitessier, ils sont des milliers à s'être élancés vers le grand large, à larguer les amarres pour entamer un long voyage, de quelques mois ou de plusieurs années, relevant à la fois d'un projet familial, d'une quête de liberté, d'une envie de "nature» et de rencontres, d'une vie alternative en somme, à la croisée des déplacements touristiques, des mobilités résidentielles et des migrations d'agrément. Chaque année, plus d'un millier de voiliers quittent ainsi les ports européens pour traverser l'Atlantique à destination des Antilles, et il serait environ 12000 à naviguer dans toutes les mers du monde, dont la moitié en Atlantique (Gaugue, 2014). Pour autant, ces flux paraissent extrêmement confidentiels, voire anecdotiques au regard des centaines de millions de migrants ou du milliard et demi de touristes 
internationaux recensés à travers le monde, alors pourquoi porter un intérêt scientifique à ce type de mobilité ?

3 Pour marginales qu'elles soient, ces mobilités questionnent les catégorisations classiques qui tendent à cloisonner de manière étanche des déplacements selon des intentionnalités distinctes. Bien plus, elles produisent des spatialités nouvelles, faites de circulations croisées à différentes échelles et selon des temporalités variables, dessinant des territorialités mouvantes. Au fond, en déroulant des itinéraires dictés par des motifs variés et emmêlés, qui combinent différents pas de temps et d'espace, scandés par l'alternance de phases de mouvement et de relative sédentarité, les plaisanciers au long cours nourrissent ce nouveau paradigme mobilitaire qui semble caractériser les sociétés contemporaines et sur lequel cet article propose d'apporter un éclairage. Ainsi, après avoir mis en lumière la fluidité des mobilités contemporaines, par-delà les catégorisations existantes, il s'agira de montrer dans un second temps combien les mobilités des plaisanciers révèlent de nouveaux modes de circuler et dessinent aujourd'hui des spatialités complexes et hybrides. De manière dialectique, le troisième temps de la réflexion permettra de découvrir qu'en jetant l'ancre en Polynésie, les plaisanciers ne font qu'inventer une nouvelle déclinaison de l'habiter mêlant de façon très intriquée circulation et ancrage.

4 Le focus qui est proposé ici sur la navigation en Polynésie française n'est pas fortuit. Outre le clin d'œil à Moitessier qui a fini par jeter l'ancre dans cet archipel du Pacifique, celui-ci est considéré par la plupart des plaisanciers comme la fin du voyage - dans les deux sens de l'ultime escale et de la finalité -, et permet ainsi de questionner de manière particulière les échelles spatiales et temporelles de la circulation et de l'ancrage. On le pressent ici, dès l'annonce du titre et à la suite de ces premières phrases introductives, le terme "ancrage» sera abondamment utilisé dans cette réflexion, au sens propre puisqu'il y sera question de navigation et d'escale, et au sens figuré de l'arrêt plus ou moins prolongé de la mobilité. L'usage de plus en plus répandu de la métaphore de l'ancrage dans les travaux en sciences sociales interroge, comme le souligne l'article très stimulant de Bernard Debarbieux sur les métaphores de l'enracinement, de l'ancrage et de l'amarrage (2014). Reprenant le questionnement d'Isabelle Lefort, il se demande en effet si l'usage récurrent et souvent impensé de la métaphore dans l'écriture géographique ne représente qu'une "facilité langagière » à des fins pédagogiques, ou bien, plus fondamentalement, constitue une «figure opératoire» (cité par Debarbieux, ibid., p. 69). Nous ne développerons pas cette discussion ici, mais il nous semblait néanmoins important de pointer cette question qui traverse, en filigrane, l'ensemble de notre propos et qui mériterait sans doute une analyse plus poussée.

5 Cet article s'appuie sur une enquête conduite sur l'île de Raiatea, en Polynésie française. Ile principale de l'archipel des Iles-sous-le-Vent, Raiatea est la seconde île la plus peuplée derrière Tahiti avec une population d'environ 12500 habitants au dernier recensement et concentre la plupart des services publics de l'archipel (collège, lycée, gendarmerie, hôpital, etc.). Nous avons choisi cette île comme terrain d'étude car elle se présente comme LA base nautique de la Polynésie, avec deux marinas qui abritent non seulement la plupart des plaisanciers au long cours en escale sur ce territoire, mais également les principales compagnies charters qui organisent des croisières à la voile au départ de Raiatea et à destination des différents archipels polynésiens. Ce travail de recherche dessine de fait un schéma mobilitaire fortement orienté par la construction 
d'une territorialité française en Outre-mer, autrement dit assumant une dimension francophone qui peut limiter partiellement la portée de cette recherche. Au total, 13 entretiens ont été réalisés sur l'île de Raiatea auprès de plaisanciers français installés sur leur bateau dans l'une des deux marinas de l'île ou logeant dans une maison. Individuels (10) ou en couple (3), les entretiens ont été menés auprès de 6 hommes et 10 femmes entre 35 et 80 ans, voyageant en couple et pour certains (4 enquêtés) en famille avec leurs enfants âgés entre 1 et 12 ans à l'arrivée à Raiatea. Au moment où nous avons mené nos entretiens, les personnes que nous avons rencontrées étaient parties de France depuis plusieurs années (de quelques années à plus de 20 ans).

6 S'ils n'ont pas toujours un capital économique élevé (la plupart d'entre eux ont tout vendu en France pour acheter leur voilier et partir en mer), ils possèdent en revanche un capital social et culturel important. Nombre d'entre eux ont fait des études et les savoirs et compétences qu'ils ont acquis tout au long de leur vie leur permettent de s'adapter rapidement dans les lieux où ils font escale. La plupart (9) ont créé leur société en arrivant sur l'île (voile, menuiserie, skipper, vente, restauration, etc.) et s'en sortent relativement bien. Les autres plaisanciers sont retraités, infirmiers, salarié et maître de conférences en disponibilité.

7 Les données mobilisées sont issues d'entretiens réalisés lors de trois missions de terrain différentes, en 2015, 2018 et 2019. Durant les deux premières nous avons conduit nos entretiens à partir d'une grille comportant quatre thématiques dont la première concernait « la trajectoire de vie », avant l'arrivée dans l'île, et la seconde « la vie dans l'île ». Dans le récit des plaisanciers, la place accordée au voyage en bateau était particulièrement importante et la précision des réponses nous a permis d'identifier chaque escale, leur durée, ainsi que les activités qui y ont été réalisées. A partir de ce matériau, nous avons cartographié les parcours des plaisanciers. De la même manière, à travers la thématique "la vie dans lîle », nous avons cherché à connaître les lieux pratiqués par les plaisanciers sur l'île et en dehors, la fréquence de ces pratiques et les activités déployées. A partir des réponses, nous avons réalisé une seconde série de cartes représentant les espaces de vie des plaisanciers en escale à Raiatea. Lors de la troisième mission toutes les cartes ont été présentées aux enquêtés afin qu'ils puissent les corriger, les compléter et les valider. Trois d'entre-elles, représentant les parcours migratoires de Simon $^{1}$ (carte 1) et de Marie (carte 2) ainsi que l'espace de vie de Michelle (carte 4), parce qu'elles assemblent de manière complexe un grand nombre de paramètres et d'échelles, ont été choisies dans cet article pour illustrer notre démonstration.

\section{Des « mobilités liquides »}

8 Notre monde est plus que jamais devenu un monde où la mobilité est reine. Omniprésente, "paradigme central de nos sociétés et de leurs imaginaires » selon C. Mincke et V. Kaufmann (2017, p. 1), elle imprègne les discours des décideurs, se déploie sur les affiches publicitaires qui vantent les voyages et le mouvement, exprime le dynamisme, la jeunesse et le bonheur, fait rimer fluidité et créativité, accessibilité et liberté. Même le programme des Nations-Unies (PNUD) exhorte les gouvernements du monde entier à "lever les barrières " pour libérer la mobilité, considérée comme un véritable moteur de développement humain. Parée de toutes les vertus, la mobilité semble constituer aujourd'hui la nouvelle doxa des sociétés néolibérales (Boltansky \& 
Chiapello, 1999), « comme un principe cardinal [...] de l'activité humaine en général et de l'organisation de la société contemporaine en particulier » (Borja, Courty, Ramadier, 2014, p. 1).

\section{Le tournant mobilitaire}

9 Elle est d'ailleurs à ce point ancrée dans nos imaginaires et modes de vie qu'on la définit le plus souvent au singulier. Pourtant, «à chaque usage situé dans l'espace et dans le temps de la mobilité correspond en fait des histoires et des usages d'une acception de cette mobilité » (ibid.). Si ces auteurs s'accordent à mettre à jour «trois mobilités en une seule ", relevant d'une triple construction artistique, intellectuelle et politique, le monde actuel est traversé et animé d'une infinité de mobilités de toute nature et à toutes les échelles, renvoyant à des imaginaires aux contours flous : touristes, navetteurs, migrants, aventuriers, travailleurs saisonniers, marins, congressistes, routiers, turbo-profs, snowbirds ${ }^{2}$, hommes et femmes d'affaires, plaisanciers, backpackers, automobilistes, étudiants des programmes d'échange, etc., participent de cette " planète nomade » (Knafou, 1998), où ils s'ignorent, se frôlent dans des circulations croisées, emboîtées ou parallèles, voire se rencontrent, parfois. Nos vies seraient ainsi devenues des «vies liquides" pour reprendre la pensée de $Z$. Bauman $(2000,2013)$, traversées par le flux incessant de la mobilité et de la vitesse.

Depuis une dizaine d'années, cette inflation mobilitaire s'est illustrée en sciences sociales par l'explosion de publications, revues, colloques, think tanks, réseaux et centres de recherche participant de l'avènement d'un mobility turn, théorisé par Sheller et Urry (2006). Selon ces auteurs, la mobilité serait devenue la clé de lecture et d'analyse principale des dynamiques économiques, sociales et politiques contemporaines. C'est ainsi que de nouveaux champs de recherche se sont progressivement construits autour de l'étude des différentes formes de mobilités (Urry, 2007), leur évolution, la manière dont elles structurent les groupes, les classes et les individus, le rôle qu'elles jouent dans la production de spatialités protéiformes, etc. (Kesselring, 2006 ; Hui et al., 2008 ; Lassen et al. 2006 ; Larsen et al., 2006).

11 Pour autant, des voix critiques commencent timidement à ébrécher cette apologie mobilitaire, en réintroduisant notamment les vertus de l'immobilité, en revalorisant les notions d'ancrage et de proximité tout en soulignant dans le même temps l'importance des rapports de pouvoir qui se jouent dans l'accès, voire le droit à la mobilité (Hannam et al. 2006, Feildel \& Martouzet 2012, Cailly 2014, Mincke \& Kaufmann 2017). La mobilité renvoie indiscutablement à des processus socio-spatiaux et exprime de façon souvent aigüe des situations de justice ou d'injustice spatiales, de relégation ou d'intégration, comme l'avait théorisé en son temps l'Ecole de Chicago en contexte urbain. C'est ainsi par exemple que des situations d'exclusion et de marginalisation sociales peuvent se lire à l'aune de la capacité ou non des individus à se mouvoir, que ce soit dans le cadre d'une mobilité imposée, permanente et quotidienne, comme dans le cas des SDF urbains (Zeneidi, 2002), ou au contraire dans une immobilité contrainte, comme les populations pauvres de certains territoires ruraux (Bonerandi, 2004), ou bien les deux à la fois comme c'est le cas pour les migrants contemporains qui alternent des phases de circulation et des phases d'immobilité forcée sous l'effet des politiques migratoires dissuasives (Weber, 2009). La mobilité se définit donc en termes de potentiel, de choix et de non choix, mais aussi de capital pour reprendre les analyses chères à Pierre 
Bourdieu en participant d'un « habitus mobilitaire » (Allemand, 2004), que l'on pourrait définir à la fois comme une capacité à se déplacer et comme un choix issu d'une stratégie ou d'un projet individuel.

\section{Repenser les catégories de mobilité}

12 Par ailleurs, la multiplication et l'intensification des mobilités a conduit à produire des catégorisations relativement étanches, le plus souvent fondées sur des critères de durée et/ou d'intentionnalité. Le tourisme se définit ainsi comme « les activités déployées par les personnes au cours de leurs voyages et de leurs séjours dans les lieux situés en dehors de leur environnement habituel pour une période consécutive qui ne dépasse pas une année à des fins de loisirs, pour affaires et autres motifs » (OMT, 2012). Une définition qui le distingue a priori de la migration qui implique un changement de domicile, dans un rayon qui peut être local, régional ou international, pour une durée longue ou définitive.

13 Commodes en apparence, ces deux catégories se trouvent pourtant de plus en plus dépassées face à l'émergence de nouvelles formes de mobilités, hybrides et intriquées, qui s'incarnent notamment dans le développement des migrations d'aménités ou d'agrément (Martin, Bourdeau \& Daller, 2012), des lifestyle migrations ou des mobilités post-touristiques. De plus en plus de chercheurs s'attachent aujourd'hui à explorer ces mobilités d'un nouveau genre, ne relevant ni tout à fait du tourisme, ni tout à fait de la migration, mais finalement des deux à la fois et qui, pourtant, traduisent des évolutions sociétales qui ne sont pas mineures (Duncan, Cohen \& Thulemark, 2013). Michaela Benson et Karen O'Reilly (2009) furent sans doute les premières à formaliser l'émergence des lifestyle migrations, qui recoupent des mobilités plurielles dans leurs échelles et dans leurs temporalités, à l'interface entre migration résidentielle et désir de re-création pour reprendre la formule de l'équipe MIT, mais qui semblent avoir en commun la recherche d'une "belle vie»: "What is revealed is the singularity of a fascinating phenomenon sharing several important themes in common, albeit with disparate threads, with the common pursuit of the 'good life' uniting these lifestyle migrants " (ibid., 2009, p. 3). Dans une même perspective, Luc Vacher (2004) questionne les contours de ces nouvelles mobilités à travers ses travaux sur le big trip des retraités australiens qui, après avoir vendu leur maison, sont nombreux à partir sur les pistes australiennes pour de longues itinérances en caravane. Ces big trippers tendent ainsi à se transformer en greynomads dans ce pays où l'identité s'est construite autour du mythe du voyageur. Comme en écho, les recherches menées par Brenda Le Bigot (2017) sur les hivernants au Maroc et les backpackers en Thaïlande soulignent bien l'originalité de ces deux formes de mobilités qui relèvent des "privileged mobilities " (Croucher, 2012). Très différentes l'une de l'autre, tant dans l'âge des voyageurs, la motivation, les itinéraires, les durées, les pratiques et les modes d'habiter, elles traduisent l'émergence de modes de vie nomades pour " aller chercher ailleurs un quotidien enchanté » (ibid. p. 487) et révèlent l'aporie des catégorisations classiques de mobilités.

14 L'ensemble des recherches réalisées dans ce champ montrent ainsi non seulement la porosité des catégories de mobilités, mais surtout l'existence, pour nombre de trajectoires de vie, d'un véritable "continuum mobilitaire» allant du déplacement touristique court et temporaire à la migration résidentielle post-touristique en passant 
par différentes formes d'expériences touristiques, de mobilités professionnelles, de circulations entre résidence principale et secondaire, etc.

\section{Habiter (la mer) : entre ancrage et mobilité}

15 Au-delà, ces mobilités singulières re-questionnent la notion d'habiter à travers la dialectique de l'ancrage et de la mobilité. Si pendant longtemps habiter a signifié résider, aujourd'hui de nombreux travaux (Lazzarotti, 2006, 2012, 2014 ; Kaufman 2008) démontrent au contraire que non seulement la mobilité est une composante essentielle de l'habiter, mais qu'elle n'est de surcroît pas du tout incompatible avec l'immobilité avec laquelle elle s'articule étroitement. L'habiter se définirait alors à travers la combinaison dialogique de la mobilité et de l'immobilité, produit du passage incessant de l'une à l'autre, que ce soit dans des pas de temps quotidiens ou dans des temps plus longs (Belton-Chevallier, 2015), où l'existence de l'une serait conditionnée par le déploiement de l'autre, et inversement (Gallez \& Kaufman, 2009).

Saisir la mobilité nécessiterait donc de prendre en compte l'ancrage, dans ses multiples points de fixation spatiaux et temporels à partir desquels s'attachent les fils du mouvement ; à l'inverse, l'ancrage ne saurait être considéré autrement que comme la finalité de la mobilité. De nombreux exemples illustrent cette dialectique. Kaufman (2008) montre par exemple que l'usage de plus en plus généralisé des transports rapides traduit certes une hypermobilité mais qui, dans nombre de cas, permet de maintenir précisément différentes formes d'ancrage, d'ordre résidentiel, familial, professionnel, etc. C'est le cas de certaines élites professionnelles qui, pour hypermobiles qu'elles soient, n'en sont pas moins très attachées à leur lieu de résidence que les moyens de (télé-)communication rapides et performants leur permettent de relier facilement. Dans leurs études sur les espaces périurbains, Feildel \& Martouzet (2012) ou encore Berroir et al. (2017) soulignent également que les navettes et mouvements pendulaires qui rythment le quotidien de ces populations constituent précisément une modalité de leur ancrage périphérique, faisant de l'habiter périurbain un condensé de l'enracinement et du mouvement.

Dans un tout autre contexte géographique et temporel, les travaux sur les espaces circulatoires et transnationaux des migrants considèrent dans bien des cas la mobilité comme la condition sine qua non de la pérennisation des ancrages sociaux et familiaux dans les territoires d'origine. A petite échelle, Giorgia Ceriani-Sebregondi (2003) évoque cette intrication de la mobilité et de l'immobilité dans son étude sur l'habiter des migrants qu'elle résume à travers la notion de "spatialité dynamique». Selon elle, celle-ci se définit comme une combinaison de territorialisation et de mouvement, de formes de fixation plus ou moins durables (en ville, dans des camps, des îles) et de projection permanente vers l'extérieur, que ce soit vers le lieu de départ avec lequel ils entretiennent des liens étroits (envoi d'argent, d'informations, etc.) ou vers le lieu suivant dans leur itinéraire.

Finalement, à des échelles variées et dans des contextes géographiques, sociaux et économiques distincts, toutes ces approches s'inscrivent dans une réflexion large questionnant la manière dont la mobilité géographique des individus modifie profondément leur rapport à l'espace, aux liens et aux lieux, et définit de nouveaux modes d'habiter, dits " 'poly-topiques', qui assemblent d'une nouvelle manière mobilité 
et immobilité et intègrent d'une autre façon la mobilité dans les conduites de vie » (Stock, 2015, p. 429).

Certaines formes d'habiter combinent toutefois de manière plus singulière l'ancrage et la mobilité, comme celles des camping-caristes français au Maroc ou des big trippers australiens évoqués précédemment qui, de fait, ont en commun de se déplacer avec «leur maison sur le dos». Il en est de même pour les marins qui habitent la mer sur leur bateau, faisant de celui-ci à la fois leur mode de transport et de résidence. Quelques chercheurs ont mis en évidence à la fois la fluidité des parcours et l'originalité de l'habiter marins, que ce soit Camille Parrain à travers ses travaux sur les territoires de haute-mer construits par les navigateurs à voile (2010) ou encore Anne Gaugue $(2011,2013,2014,2018)$ qui a étudié ceux qu'elle nomme «les plaisanciers au long cours ». Le camping-car, la caravane et le voilier sont donc des lieux nomades qui abritent paradoxalement une sédentarité exacerbée à l'origine de micro-territorialités particulièrement bien étudiées par Stéphanie Josso dans sa thèse d'anthropologie (2010). « Grâce à leur maison flottante, écrivait Anne Gaugue à propos des plaisanciers au long cours, ils voyagent de port en port et découvrent de nouveaux lieux, tout en étant chez eux» (2014, p.1). Au fond, habiter en mer interroge de manière paradigmatique la dialectique de l'ancrage et de la mobilité dans l'unité de lieu que constitue le bateau.

Plus largement, l'étude que nous proposons ici sur les plaisanciers au long cours offre un prisme intéressant et insolite permettant de saisir l'hybridité des mobilités, entremêlant tourisme et migration, immobilité et mouvement, courtes et longues distances, déplacement définitifs et provisoires. Il s'agira de questionner de façon critique la nature et la finalité de celles-ci, leurs modes de catégorisation et leurs liens à l'ancrage, dans des univers géographiques résolument liquides.

\section{Prendre le large : des mobilités plurielles et profondément hybrides}

\section{Partir en mer : entre projet de vie et voyage fortuit}

21 La majorité des plaisanciers que nous avons rencontrés ont parcouru les mers du globe, alternant navigation et escale, pendant plusieurs mois, voire plusieurs années, avant d'arriver dans la marina d'Uturoa ${ }^{3}$. Leurs récits soulignent l'hétérogénéité des trajectoires mais surtout la grande variété des ressorts du départ. D'une part, les raisons diffèrent d'une personne à l'autre et d'autre part, pour un même individu, celles-ci sont multiples et enchevêtrées. Pour autant, on distingue grosso modo deux types de situations : il y a ceux dont le voyage est un projet de vie construit et préparé sur plusieurs années et ceux dont le voyage est plus fortuit, relevant davantage de l'opportunité, de l'occasion ou de la réaction à un évènement (plus ou moins difficile à vivre).

D'après nos entretiens, le premier groupe est constitué de plaisanciers qui partent au moment de la retraite ou dont le départ est motivé par la volonté de voyager et de découvrir le monde, que cette envie soit un rêve d'enfance ou qu'elle se soit progressivement construite au cours de la vie et des expériences vécues. C'est le cas, par exemple, de ce plaisancier qui nous explique comment est née son envie de partir : 
"C'est parce que mon père avait un voilier. Du coup on faisait des balades en mer le week-end; donc c'est comme ça que je me suis dit: 'tiens c'est pas mal le bateau pour voyager !'. Après je suis parti avec un copain qui avait un petit bateau, on est allé jusqu'aux Canaries et là je me suis dit: 'ah ouais c'est pas mal ça !' et je me suis dit 'allez hop je reviens en France, je m'achète mon bateau et je pars' » (Paul, 48 ans)

Le deuxième groupe concerne plutôt des personnes qui partent suite à un évènement imprévu ou un accident de vie comme une rupture professionnelle par exemple ou encore le décès d'un proche. Ce départ peut être également de l'ordre du hasard, l'opportunité d'acheter un bateau, etc.

«On était à Marseille et en fait c'est des raisons familiales... enfin moi c'était la perte de mon père qui m'a fait réaliser qu'il fallait profiter maintenant avant qu'il soit trop tard, lui il a aussi perdu son père [...] on avait un voilier donc on est parti pour faire le tour du monde en voilier " (Lisa, 35 ans)

Pour autant, quelle que soit la, ou même, les raisons qui poussent les plaisanciers à prendre le large, l'envie de liberté et le rejet des contraintes se retrouvent dans la quasi-totalité de nos entretiens, comme l'illustre l'extrait suivant :

« Non, c'est un tout, finalement le temps de navigation est très court par rapport au temps des escales. Donc c'est juste le fait de pouvoir faire ce qu'on veut, sans contraintes. Des contraintes on en a toujours, mais elles sont plus liées directement aux choix qu'on fait » (Bernard, 50 ans)

\section{Parcourir les mers : des circulations à géométrie variable} départ de l'Europe, qui sillonnent les Antilles quelque temps, puis empruntent le canal de Panama pour rejoindre le Pacifique et atteindre la Polynésie. Cet itinéraire, que l'auteure présente de manière relativement linéaire, se révèle plus complexe dans les faits. Les entretiens que nous avons menés à Raiatea nous permettent en effet de corroborer les contours de cette trajectoire-type tout en l'affinant grâce à des focus à différentes échelles spatiales et temporelles. effectivement deux itinéraires représentatifs de spatialités multiscalaires produites par la majorité des plaisanciers enquêtés. Partant de France métropolitaine après avoir navigué en Méditerranée, les navigants rejoignent le bassin caribéen après quelques arrêts aux îles Canaries et au Cap Vert. Puis, après avoir circulé dans la Caraỉbe, ils traversent le canal de Panama, s'arrêtent aux îles Galápagos avant de rejoindre les îles Marquises en Polynésie française. Si elle met en évidence une trajectoire que nous qualifierons de « classique ", cette carte montre aussi dans le détail que le trajet est loin d'être linéaire. Le chemin parcouru par les plaisanciers depuis l'Europe jusqu'en Polynésie française apparait en effet entrecoupé de phases de circulation et de phases de sédentarisation qui se déclinent à différentes échelles et sur des périodes allant de quelques semaines à plusieurs années. 
Carte 1. Le parcours migratoire de Simon

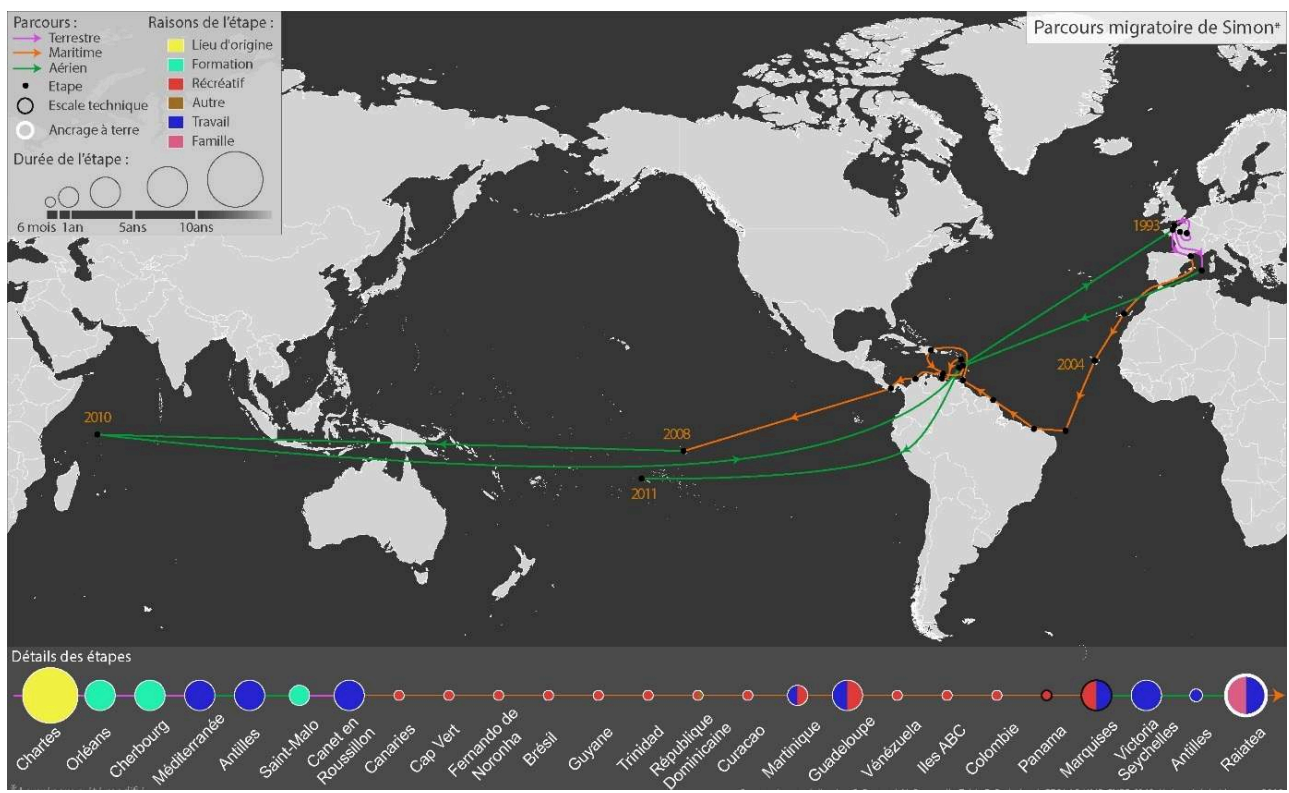

Source : Entretiens de terrain

Carte 2. Le parcours migratoire de Marie

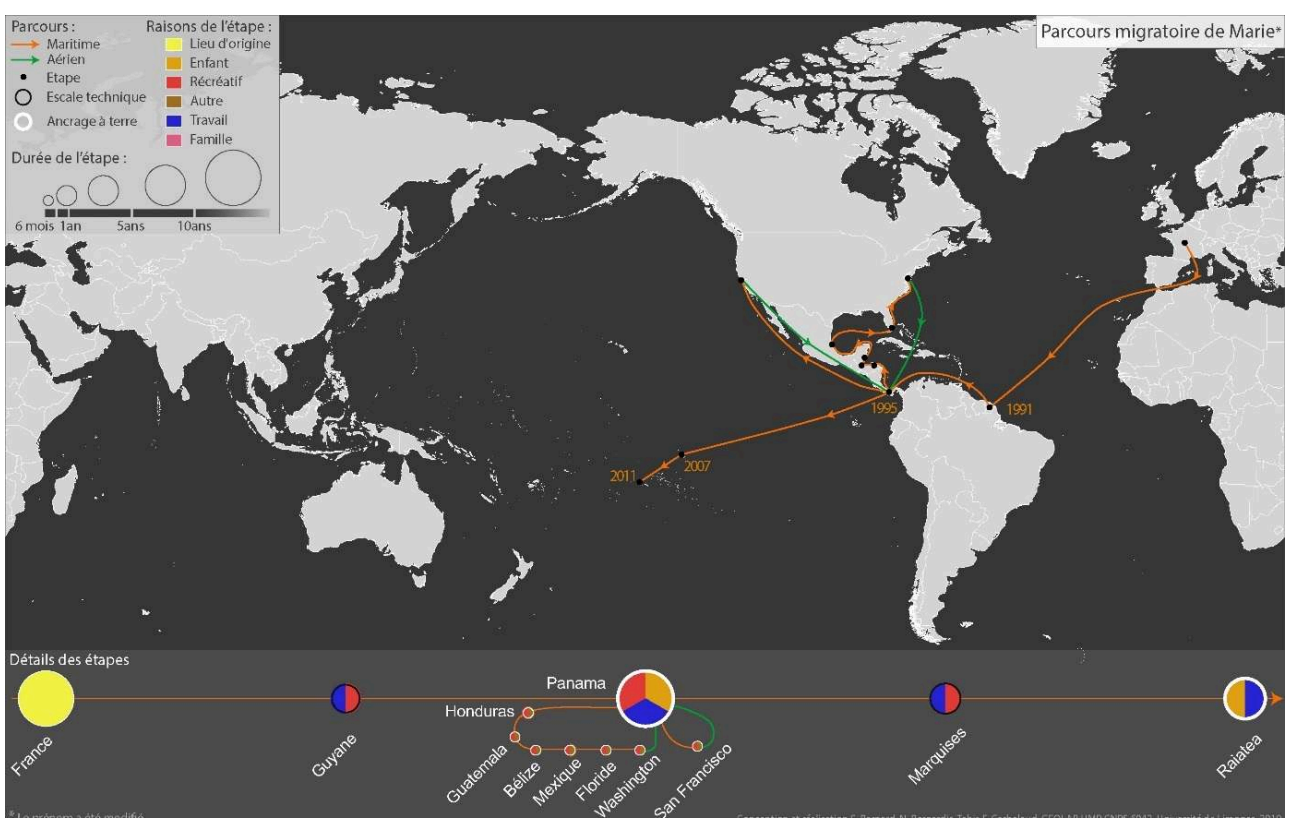

Source : Entretiens de terrain.

Ces différentes séquences enchâssées dans la trajectoire linéaire des plaisanciers peuvent prendre plusieurs formes: des va-et-vient ou circulations à rayon géographique limité entre plusieurs territoires d'une même région, ou bien des allersretours ponctuels vers des destinations plus ou moins lointaines. Les premières sont repérables sur les cartes par la concentration de mobilités courtes sur une petite zone et sont particulièrement nombreuses dans les bassins méditerranéen et caribéen, propices à une circulation concentrée. 
«On est parti en 2004 et on est arrivé en 2008 en Polynésie. Donc évidemment... oui : Canaries, Cap Vert, Brésil, Guyane, Trinidad, toutes les îles, Colombie, Venezuela, Panama. [...] On s'est arrêté aux Antilles, pendant un an et demi à peu près,[...] et on est reparti donc avec le gros bateau qu'on a acheté en République Dominicaine ; on a refait un peu le même parcours, Antilles et Amérique du Sud et on a passé le canal de Panama et on est arrivé en 2008 aux Marquises » (Simon, 45 ans)

28 A l'échelle du bassin caribéen, les individus paraissent hyper mobiles, circulant d'une île ou d'un territoire à l'autre pour des séjours de quelques semaines voire quelques mois, mais rarement davantage. En revanche, à plus petite échelle - celle de leur voyage -, leur passage par les Antilles s'apparente à une pause et prend la forme d'une sédentarisation temporaire dont ils ne connaissent pas, a priori, la durée. Les plaisanciers ne se contentent donc pas de traverser ces régions : ils y restent souvent quelques années, circulant d'un territoire à l'autre.

Le chemin parcouru par les plaisanciers depuis l'Europe jusqu'en Polynésie française peut être également entrecoupé d'allers-retours réalisés à différents stades du parcours. Ceux-ci peuvent être effectués en avion uniquement ou bien associer avion et bateau. Le parcours migratoire de Simon, illustré par la carte 1 , montre qu'après être arrivé en Polynésie française, il a effectué un aller-retour aux Seychelles où il a séjourné pendant un an avant de revenir plus tard à Raiatea. L'emboîtement d'allersretours avion/bateau survient, par exemple, à l'occasion du convoyage d'un bateau, pratique fréquemment réalisée par les plaisanciers :

«On a fait le tour par la Patagonie, une partie donc du Chili. Entre temps en fait, en Patagonie, on a rencontré un bateau et on nous a demandé si on pouvait convoyer ce bateau là jusqu'en France. Donc en fait nous on a posé notre bateau dans un port du Chili et on a convoyé ce bateau de Rio au Brésil jusqu'à Marseille » (Annabelle, 40 ans).

30 Après avoir convoyé le bateau, cette femme et son mari sont retournés au Chili en avion pour récupérer leur voilier et poursuivre leur voyage. De manière générale, nombreux sont les plaisanciers qui articulent leur voyage à la voile avec des circulations en avion. L'une d'entre elle (Camille, 35 ans) est arrivée aux Antilles en avion puis a continué sa route vers la Polynésie française en bateau, après avoir circulé quelques années dans le bassin caribéen. A l'inverse, deux autres plaisancières (Lisa, 35 ans et Michelle, 55 ans) sont arrivées aux Antilles en bateau via la route "classique » des plaisanciers et sont parties en Polynésie française en avion.

31 Pour finir, il faut également préciser que certains plaisanciers, plus rares, dessinent des trajectoires atypiques en empruntant des routes plus difficiles, comme celle du Cap Horn par exemple :

«Au début on a traversé l'Atlantique, on s'est retrouvé au Brésil on a passé pas mal de temps là-bas parce qu'on connaissait des gens qui étaient là-bas. Et en fait on a décidé du coup de pas prendre la route normale mais de passer par le sud, par la Patagonie» (Annabelle, 40 ans).

D'autres composent leur trajectoire au fur et à mesure du voyage. L'une d'entre elles a ainsi navigué jusqu'au sud de l'Amérique mais n'a pas passé le cap Horn compte tenu des difficultés de navigation. Elle a dès lors décidé de remonter le long des côtes de l'Amérique du sud pour circuler quelques temps dans la Caraïbe avant de passer le canal de Panama. 
On le voit bien, les parcours des plaisanciers ne sont ni linéaires, ni homogènes, mais entrecoupés de phases de circulations et de phases de sédentarisation plus ou moins longues dans l'espace et dans le temps. Au fond, cette manière de concevoir et de saisir l'articulation fine entre ancrage et mobilité nous renvoie à la dialectique des spatialités et des territorialités, les premières exprimant les circulations des plaisanciers qui dessinent et agencent l'espace géographique maritime, les secondes correspondant aux processus de construction sociale et d'appropriation des lieux qui jalonnent ces routes. Cette combinaison dialogique de la mobilité et de l'immobilité des plaisanciers pendant leur voyage fait écho à d'autres associations contraires et néanmoins complémentaires, théorisées dans d'autres contextes anthropologiques. La métaphore de l'arbre et de la pirogue chère à Joël Bonnemaison en est une, résumant de manière extraordinairement précise et imagée toute l'histoire des peuples océaniens fondée sur l'amalgame de l'ancrage et du mouvement, le bois des pirogues sillonnant en tous sens le Pacifique provenant des arbres profondément enracinés dans la terre (1996). De même, dans les années 1970 nous rappellent Debarbieux et al. (2008), certains géographes anglophones ont formalisé un autre couple conceptuel : space et place. «Le concept de space leur a permis d'appréhender l'espace comme un champ sillonné de flux quand le concept de place visait à rendre compte des processus par lesquels les individus et les collectifs construisaient des formes d'attachement affectif ou symbolique » (ibid., p. 76).

ent, ces circulations plaisancières questionnent et illustrent la manière dont les mobilités non seulement transforment le rapport des hommes à l'espace et aux lieux, mais, au-delà, définissent les nouveaux fondements identitaires de nos sociétés contemporaines (Cattan, 2014).

\section{Des mobilités plurielles pour des intentionnalités hybrides}

Les mobilités des plaisanciers ainsi que le choix des lieux d'escale sont par ailleurs déterminés par des motivations variées. Entre migration résidentielle, déplacement touristique ou encore mobilité professionnelle, le voyage dans son ensemble juxtapose et parfois combine plusieurs intentionnalités liées aux envies et contraintes des plaisanciers. La dimension profondément hybride du voyage peut se lire à travers le prisme des escales dont la diversité des fonctions nous permet d'interroger et de repenser les catégories de mobilités actuelles.

Sur ce sujet, Anne Gaugue (2018) avait réalisé une typologie extrêmement éclairante qui la conduisait à distinguer trois types d'escale : les escales techniques, les escales récréatives et les escales mixtes qui associent besoins techniques et envies récréatives. Pour structurante qu'elle soit, cette typologie n'épuise pas les variantes et formes hybrides d'escales qui sont ressorties lors de nos entretiens.

En dehors des raisons techniques et des aspirations récréatives qui reviennent effectivement de manière récurrente dans les discours, le besoin de travailler pour refaire une caisse de bord ${ }^{4}$ est un motif souvent entendu dans les témoignages. Les plaisanciers choisissent en effet leurs escales en fonction des opportunités de travail, souvent plus nombreuses ou faciles dans les territoires francophones, que ce soit à terre dans les hôpitaux, écoles, lycées, cafés, restaurants, pharmacies, etc., ou bien dans le milieu nautique lorsque l'économie de la navigation charter est développée et où les possibilités d'emploi comme skippers ou hôtesses notamment sont constamment renouvelées. 
«L'avantage d'être français, il faut bien le reconnaitre, c'est que l'on a quand même des DOM-TOM partout dans le monde et nous on navigue selon l'endroit où on va pouvoir refaire une caisse de bord, trouver du travail. Et les îles françaises sont quand même pas mal, un bon spot pour ça, et puis on en a dans chaque océan » (Camille, 35 ans) conduisant des plaisanciers à enchaîner par exemple des escales pour diverses raisons (récréatives, puis techniques, puis économiques par exemple), ou bien à combiner plusieurs finalités dans un même arrêt, comme c'est par exemple le cas dans la trajectoire de Simon où les escales en Guadeloupe et Martinique associent étroitement récréation et travail. Initialement motivés par le plaisir de découvrir de nouveaux lieux, ces arrêts lui ont en effet permis de trouver un poste de chef de base puis d'ouvrir une base nautique, deux activités qui en retour lui ont laissé la possibilité de rester dans la zone pour pratiquer des activités récréatives. De même, Marie s'est arrêtée au Panama avec sa famille pendant dix ans et nous explique que son installation avait plusieurs objectifs : scolariser ses enfants, travailler et découvrir le pays. Ils ont d'ailleurs profité de ce cadre qu'ils appréciaient beaucoup pour se sédentariser en construisant une maison et produisant leurs propres fruits et légumes. Souvent valorisée dans la littérature scientifique relative à la plaisance au long cours, la dimension récréative, on le voit, est intimement mêlée à d'autres motivations, voire remise en question par certains plaisanciers qui soulignent leur implication active dans l'éducation des enfants, en assurant notamment leur scolarisation à bord, et qui de ce fait ne se sentent pas du tout dans une démarche touristique.

L'exemple des convoyages est très intéressant pour mettre en lumière cette hybridité du voyage. Les deux allers-retours réalisés par Marie entre Panama et Washington puis entre Panama et San Francisco traduisent bien la combinaison des motivations. Si la première destination avait pour objectif de vendre son bateau et la seconde était un convoyage, ces deux expériences étaient aussi l'occasion pour elle de découvrir de nouveaux rivages. Lors du second voyage, elle en a même profité pour rester travailler à San Francisco pendant un mois et demi. 
42 Les mobilités des plaisanciers sont bien l'aboutissement d'une combinaison de motivations (touristiques, professionnelles, médicales, éducatives, etc.) et prouvent la porosité des catégories établies dans la littérature. D’ailleurs, nous avons rapidement mesuré les limites de cette segmentation arbitraire au début de notre enquête lorsque les plaisanciers ne parvenaient pas à démêler les raisons de leurs escales. Mais c'est aussi l'existence d'un continuum mobilitaire que cette étude met à jour : une mobilité touristique peut se transformer en migration résidentielle et/ou professionnelle, avant de retrouver un peu plus tard une finalité à nouveau récréative. Finalement, hybridation des intentions et continuum des spatialités se confondent pour produire une mobilité aujourd'hui profondément syncrétique, qui bouscule des lignes scientifiques et épistémologiques trop longtemps cloisonnées, inopérantes aujourd'hui pour saisir ce nouveau paradigme sociétal.

43 Si l'exemple des plaisanciers faisant voile vers la Polynésie illustre ainsi particulièrement bien diverses modalités d'ancrage déployées au fil de leurs mobilités, leur longue escale à Raiatea nous permet inversement de saisir, à une autre échelle, comment leur amarrage génère de nouvelles circulations.

\section{Jeter l'ancre à Raiatea : la fin du voyage?}

\section{La Polynésie française : un mythe pour les plaisanciers ?}

Comme nous l'avons souligné plus haut, partir en mer n'est pas toujours un projet construit et programmé à l'avance. Le choix de l'itinéraire non plus, se structurant le plus souvent au fur et à mesure du parcours. Ils sont bien peu nombreux en effet à savoir pour combien de temps ils partent et quelle sera la date de retour. «Il a juste dit à ce moment-là qu'on partait pour trois mois, et voilà ça fait 10 ans! Tout ça pour dire que c'était pas du tout calculé» (Annabelle, 40 ans). De même le choix des routes et des escales s'établit au fil de l'eau. En est-il de même pour la Polynésie française ? Est-ce une destination comme les autres qui émerge au fur et à mesure de l'avancée du voyage, ou bien tient-elle une place à part dans l'esprit et les représentations des plaisanciers, pétris comme ils le sont pour la plupart des récits de grands navigateurs qui ont définitivement jeté l'ancre en Polynésie?

Dans son article, Anne Gaugue révèle des témoignages qui montrent que la Polynésie française détient une place particulière dans le parcours migratoire des plaisanciers :

"La Polynésie est une destination qui fait rêver les plaisanciers hauturiers, et beaucoup considèrent cette étape comme un des moments forts de leur navigation. Dans l'entre-deux-guerres, la lecture des romans de Melville ou de Stevenson a nourri les rêves de Polynésie des pionniers du voyage maritime d'Alain Gerbault à Ella Maillart » (Gaugue, 2014)

Aujourd'hui, les témoignages que l'on peut lire sur les blogs internet continuent de faire de ce territoire un des hauts lieux de la grande croisière. "L'arrivée d'une traversée comme celle du Pacifique procure forcément de fortes sensations, mais, cerise sur le gâteau, ce qu'on trouve de l'autre côté n'est pas une île quelconque, ni une terre banale. C'est LA terre. La "Terre des Hommes" comme ils disent, la Terre sauvage, la Terre de la générosité, la Terre promise... " raconte Eric lorsque lui et sa famille arrivent aux Marquises ${ }^{5}$. Mais c'est surtout "l'effet Moitessier » qui semble fondamental pour comprendre la place de la Polynésie française dans les parcours migratoires des 
plaisanciers : la majorité d'entre eux ont lu les ouvrages du navigateur français qui acheva son double tour du monde en Polynésie française (Tahiti, Ahe et Moorea) où il vécut jusqu'à sa mort en 1994. Pour autant, alors que nous pensions que l'imaginaire puissant secrété par la Polynésie (Staszak, 2003) en faisait comme un graal pour la plupart des plaisanciers, nous n'avons pas décelé cet effet-mythe dans les différents entretiens que nous avons conduits à Raiatea.

Tout d'abord, l'escale en Polynésie française intervient après un séjour dans les îles de la Caraïbe que beaucoup décrivent de manière très mitigée, évoquant un racisme exacerbé et une société clivée qui les poussent à partir. Lorsqu'ils arrivent en Polynésie française, ils n'hésitent pas à comparer ce climat de tension avec "l'accueil jovial des Polynésiens » et le climat social « apaisé » du territoire. Par ailleurs, les images des lagons bleu turquoise, des plages de sable blanc ou encore de la «Vahine » chère à Gauguin, n'ont quasiment pas été évoquées dans les entretiens. Pour certains, la Polynésie française c'est davantage "la continuité quand on a fait la Méditerranée, qu'on a traversé l'atlantique et qu'on a navigué dans la Caraibe» (Michelle, 55 ans). Pour la plupart, c'est surtout une étape naturelle sur la route des DOM TOM, un territoire français où il est facile de trouver du travail permettant de remplir la caisse de bord et de scolariser des enfants :

«Quand on s'est arrêté à Panama et qu'on a fait le choix de venir dans le Pacifique,

il fallait qu'on trouve un lieu pour que Léo puisse faire son lycée. Y en avait pas beaucoup, on a privilégié les îles françaises, par facilité, de travail, d'échanges pour lui aussi avec des jeunes de son âge. Et parlant la même langue » (Anna, 45 ans)

La Polynésie française est finalement devenue un haut lieu de la plaisance hauturière dans le monde maritime, à la fois par la présence de nombreux lagons facilement navigables mais aussi du fait de l'implantation de nombreuses sociétés de charter. Ainsi, les plaisanciers qui ont, pour la majorité, des diplômes de navigation, obtiennent facilement du travail dans ces sociétés de charter où l'embauche est d'autant plus facile qu'il y a très peu d'habitants locaux formés pour ce type d'activité.

On le voit, le choix de faire voile vers la Polynésie ne tient pas vraiment du mythe mais d'une décision le plus souvent consciemment objectivée. Mais pourquoi l'île de Raiatea?

\section{Le choix de Raiatea : un «bon compromis » en Polynésie française}

Dans les discours des plaisanciers, la Polynésie française apparait comme un territoire polarisé autour de trois pôles : les îles Marquises qui jouent le rôle de porte d'entrée du territoire; Tahiti qui est le centre administratif, économique et permet une escale technique ; et Raiatea, qui constitue le centre de la navigation charter (carte 3). 
Carte 3. La trajectoire type des plaisanciers en Polynésie française

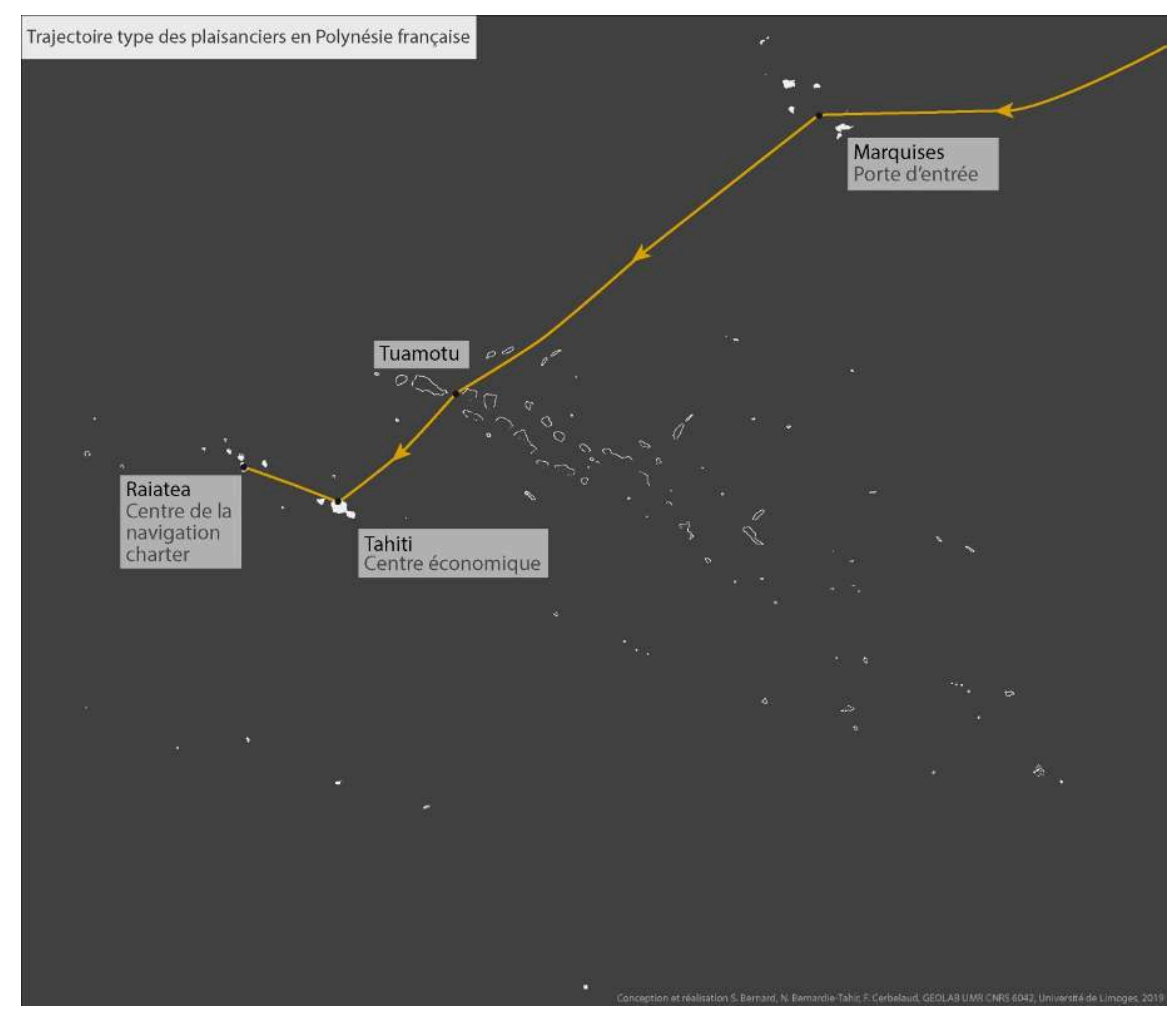

Source : Entretiens de terrain.

51 Les îles Marquises sont les premières terres visibles après une longue traversée du Pacifique depuis le Panama. La majorité des plaisanciers s'y arrêtent pour se reposer après les trois à cinq semaines de navigation. Certains y passent quelques mois pour découvrir les différentes îles de l'archipel, d'autres peuvent y séjourner quelques années alternant travail - lorsqu'ils ont des opportunités (postes au collège, hôtellerie, roulotte, etc.) -, loisirs et découverte. Le « climat paisible des îles », les paysages et « la gentillesse des Marquisiens" sont souvent évoqués dans les entretiens. Le peu de travail disponible dans l'archipel des Marquises oblige toutefois les plaisanciers à compter sur leurs ressources financières pour y rester. Généralement, le départ intervient au moment où la nécessité de remplir la caisse de bord se fait ressentir. Parfois c'est l'isolement (principalement lorsqu'il y a des enfants à bord), l'absence de structures scolaires ou hospitalières qui poussent les plaisanciers à partir vers d'autres îles.

«On a atterri aux Marquises, on a créé une roulotte crêpes et glaces, et devant les difficultés de la scolarité de Natacha (ça s'arrêtait en 2nde), on la voyait pas aller en pension à Tahiti alors que nous on restait aux Marquises. On a vendu notre roulotte et on est venu en recréer une ici [à Raiatea], parce qu'il y a un lycée qui va jusqu'au bac » (Marie, 60 ans)

52 L'arrêt dans les Tuamotu n'est pas systématique pour tous les plaisanciers, l'objectif, après les Marquises, étant surtout de rejoindre Tahiti. Cependant la voie de navigation pour rejoindre Tahiti depuis les Marquises traverse les atolls des Tuamotu et il n'est pas rare que les plaisanciers s'arrêtent quelques semaines, voire quelques mois, pour découvrir les îles de l'archipel. 
53 Tahiti reste toutefois le second objectif des plaisanciers. Capitale de la Polynésie française, centre administratif et économique, Tahiti combine plusieurs atouts invitant à faire escale. La marina Taina propose plusieurs commodités qui permettent de rafraichir et ravitailler le bateau (pompe pour les eaux noires, laverie, ateliers techniques, commerces de proximité, zone de carénage, etc.), divers loisirs comme la présence d'une plage à proximité, des centres de plongée et des restaurants/bars ainsi que l'accès à internet qui permet de prendre contact et de donner des nouvelles aux proches. Pourtant, pour la majorité des plaisanciers, le séjour à Tahiti ne dure que quelques mois. Après des semaines en mer, dans l'archipel des Marquises ou des Tuamotu, l'arrivée à Tahiti est rude, parfois même Tahiti est évité.

«Nonononon, pas de grande ville, pas de stress, pas d'embouteillage, ça aurait mieux marché, on aurait mieux fonctionné, mais non, la qualité de vie en premier » (Marie, 60 ans)

54 Les discours négatifs sur Tahiti sont très présents dans les entretiens: stress, embouteillages et insécurité sont récurrents dans les témoignages. L'ambiance au sein de la marina, ou plutôt l'absence d'ambiance, est également décevante. Située en bord de route, celle-ci est loin de correspondre aux descriptions faites par les précédents navigateurs sur lesquelles se sont construites leurs représentations. Par ailleurs, le contraste est si fort avec leurs expériences dans les archipels des Marquises et des Tuamotu qu'ils ont du mal à s'adapter à la vie métropolitaine de Tahiti.

« Je suis tombée enceinte de Lucas aux Marquises. On est allé à Tahiti, après 6 mois de Marquises, deux mois de Tuam. T'arrives à Tahiti tu fais : 'ah non, non on peut pas rester là'. Tu sors de la marina, t'es sur la RDO, la quatre-voies, on a pété un plomb. 'Vite il faut partir, c'est où le prochain hôpital ? Du coup c'était ici » (Camille, 35 ans)

Dans ces conditions, Raiatea fait figure de bon compromis, mieux équipée que les Marquises, sans les inconvénients de Tahiti. Deuxième centre économique de la Polynésie française, Raiatea abrite deux marinas, une zone de carénage, plusieurs services administratifs du pays (la direction de l'agriculture, la direction de l'urbanisme, les impôts, la direction de la santé, OPT, etc.), un hôpital, trois lycées (privé et public), plusieurs collèges, des commerces, etc. Mais parallèlement elle offre un environnement paisible et sécurisé particulièrement attractif pour les plaisanciers qui voyagent en famille.

«On préférait Raiatea de loin par rapport à Tahiti, c'est plus tranquille comme île, c'est calme. A Tahiti c'était trop compliqué, en plus avec les enfants...Là ils mènent leur vie maintenant, ils sont indépendants, autonomes, il y a pas de problèmes de sécurité, et d'organisation... voilà ils s'autogéraient, que je sois en charter et qu'elle soit au travail. Tout le monde se connait ici, ils connaissent tout le monde sur la marina » (Simon, 45 ans)

56 Par ailleurs, Raiatea étant le premier centre de la navigation charter en Polynésie française, elle attire également de nombreux plaisanciers à la recherche de travail.

« En fait oui, on s'est un peu posé à Tahiti et on est venu à Raiatea parce que c'est ici qu'il y a toutes les bases de charter, tous les catamarans de charter donc clairement pour moi, en appelant un petit peu, j'ai pu bosser tout de suite » (Annabelle, 40 ans).

On le voit, la plupart des plaisanciers font escale à Raiatea pour des raisons diverses, pour la naissance d'un enfant, pour la scolarisation au lycée, pour le travail, pour la qualité de vie, et pour tout cela à la fois dans le cadre d'intentionnalités mêlées. Pour autant, quelles que soient les motivations qui président à cette escale, l'ancrage à Raiatea ne signe pas nécessairement la fin de la mobilité pour les plaisanciers qui 
déploient dans cette île une forme d'habiter spécifique, combinant à grande échelle sédentarité et circulation.

\section{Habiter sur un bateau, entre mobilité et ancrage}

58 Dans ses travaux, Camille Parrain (2010) compare le bateau à une île flottante ou une " île voyageuse " ${ }^{6}$ pour reprendre les termes de Segalen dans Les Immémoriaux (2001, p. 91). Si l'île peut se définir par des ruptures comportementales et spatio-temporelles, celle-ci distingue quant à elle trois ruptures concernant l'objet bateau qui lui permettent de justifier la comparaison : celle du passage entre le ponton et le bateau, celle du bateau comme nouveau mode d'habiter, et celle de la traversée, considérée comme une expérience initiatique (ibid. p, 397).

La navigation en voilier concilie donc de manière très dialogique la circulation en mer et l'ancrage domestique dans le territoire de poche que constitue le bateau, produisant de fait un mode d'habiter assez original. Dans cet espace miniature et mobile, les individus, souvent en famille, déploient toutes les activités du quotidien : ils y dorment, travaillent, étudient (pour les enfants qui, encadrés par leurs parents, suivent les cours du CNED), cuisinent, bricolent, contemplent la mer, s'adonnent à toutes sortes de loisirs (maritimes ou non), produisant des territorialités resserrées pas toujours simples à articuler, comme l'a très bien montré Stéphanie Josso dans sa thèse d'anthropologie (2010).

60 Néanmoins, l'île-bateau peut également perdre sa mobilité en se sédentarisant dans un port ou dans une marina. C'est le cas pour les plaisanciers que nous avons interrogés à Raiatea, qui continuent de vivre en partie sur le bateau, tout en élargissant leurs espaces de vie à la marina et, au-delà, vers le lagon ou le reste de l'archipel. L'installation à Raiatea permet donc un mode d'habiter hybride, différent de celui qui prévaut lors de la navigation, articulant un ancrage domestique sur le bateau et dans la marina, et des circulations à différentes échelles dans l'île, que ce soit au sein du lagon de Raiatea/Tahaa, dans l'archipel des Iles Sous Le Vent ou dans toute la Polynésie française.

61 Pour la plupart des plaisanciers, la marina devient un microcosme, à la fois lieu de vie, de loisirs et parfois même de travail pour ceux qui ont un emploi dans le monde du nautisme. Ils cultivent ainsi un entre-soi très marqué, multipliant les apéros, barbecues, baignade avec les enfants en fin de journée, ou organisant des sorties communes durant le week-end. Tous admettent que l'essentiel de leur réseau social, professionnel ou amical, ne va pas au-delà du milieu nautique et intègre finalement peu de Polynésiens qui ont souvent un discours assez négatif sur ces « Français qui vivent entre eux $»^{7}$.

62 A cet amarrage exacerbé se combinent de nouvelles circulations qui se déploient toutefois dans un rayon géographique plus limité. Le bateau permet d'abord à nombre de plaisanciers de profiter du lagon de Raiatea-Tahaa et de ses motu, en particulier Iriru et Ofetaro, récemment dotés en eau potable, où ils viennent régulièrement passer une journée voire un week-end entier. Ceux qui recherchent plus de tranquillité circulent dans le lagon pour s'arrêter, un peu plus loin, sur les motu plus sauvages autour de l'île de Tahaa. Certains d'entre-deux sortent du lagon de Raiatea-Tahaa et naviguent entre les îles de l'archipel pour passer quelques jours dans d'autres lagons, 
soit pour des raisons professionnelles (navigation en charter), soit à des fins récréatives.

63 L'espace de vie de Michelle (55 ans, en couple sans enfant) illustré par la carte 4 révèle particulièrement bien ces mobilités dans l'ancrage. Sur l'île, son espace de vie s'étend du centre-ville d'Uturoa, au nord, à la vallée de la Faaroa, à l'est, et elle circule d'un bout à l'autre de cet espace essentiellement à vélo. Elle privilégie la vallée de la Faaroa, où elle vit, pour s'adonner à la lecture, à l'écriture et à la peinture et se rend à Uturoa pour faire ses courses ou pratiquer des activités telle que la gymnastique.

« Je fais du vélo, la gym avec Marie, la danse polynésienne et puis ce que j'aime c'est l'équitation, la natation voilà, après la marche aussi en fait dans mes occupations tout est basé sur ça, sur le sport parce que si tu veux il y a rien d'autre ici bon si après chez moi j'aime bien rester pour lire évidemment, je lis, et puis je dessine aussi »

64 Son travail, dans une entreprise de navigation charter, lui permet de pratiquer un second espace qui est celui de l'archipel des Iles Sous Le Vent. Régulièrement elle emmène quelques clients découvrir l'archipel à bord d'un catamaran. Ce deuxième espace de vie est donc plutôt d'ordre professionnel, mais elle précise tout de même que son travail lui permet de profiter des lieux et de pratiquer des activités récréatives :

«Je profite en même temps du bateau et je me lève très tôt pour pouvoir avoir une heure pour moi, l'après-midi pouvoir nager [...] Je vis comme le touriste tu vois, je fais mon travail mais je vis comme le touriste en même temps, je jouis du bateau et de l'environnement "

Cette plaisancière pratique enfin le territoire au-delà des frontières de l'archipel en se rendant également aux Marquises, dans les Tuamotu et aux Australes. Après avoir organisé pendant des années des croisières dans l'archipel des Marquises et des Australes, elle se rend aujourd'hui dans ces archipels pour ses vacances. En revanche, elle continue d'organiser avec son ami des séjours touristiques consacrés à la pêche dans les lagons de Rangiroa, Tikehau et parfois Mataiva dans l'archipel des Tuamotu. 
Carte 4. L'espace de vie de Michelle

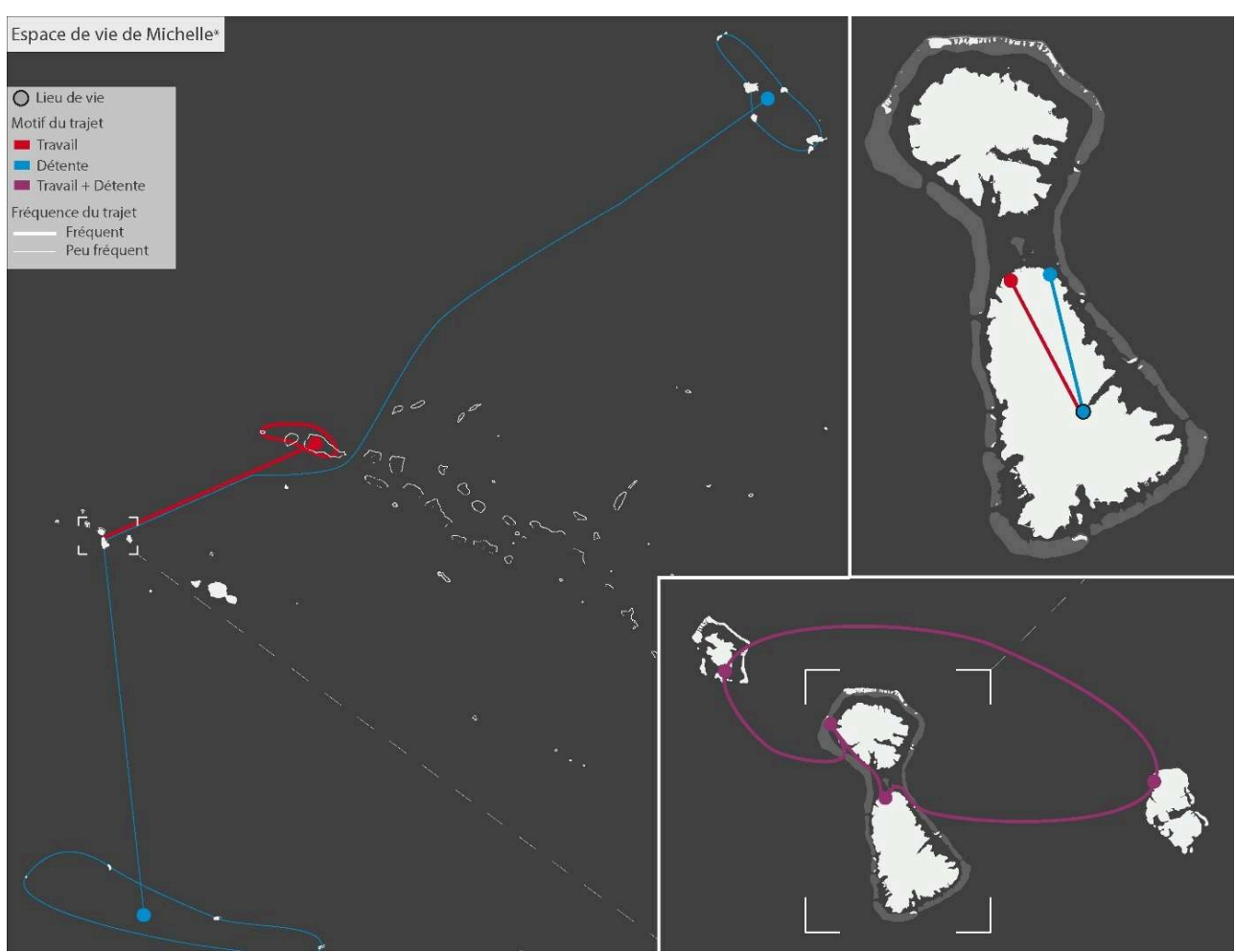

Source : Entretiens de terrain.

Elle résume d'ailleurs ainsi les contours de ce mode d'habiter original, entre terre et mer, entre mouvement et sédentarité, entre îles et archipels :

«On vit en Polynésie, c'est-à-dire nous on vit dans plein d'îles, on vit pas que sur une île [...] ça nous permet d'habiter, d'élargir notre territoire je dirais, on connait des gens ici, à Bora, Huahine, on vit pas que sur Raiatea, on vit sur un plan d'eau avec des îles, tu vois, donc ça élargit bien ton territoire ».

Finalement, à Raiatea, les plaisanciers sont tout autant ancrés dans la mobilité que mobiles dans l'ancrage.

\section{Conclusion}

En étudiant le parcours des plaisanciers au long cours depuis l'Europe jusqu'en Polynésie française et leur installation à Raiatea, cet article a révélé la singularité d'un objet géographique encore évanescent mais dont la capacité heuristique est prometteuse, et mis en lumière trois points majeurs. Le premier concerne la porosité des catégories de mobilités dont les contours, établis de manière relativement cloisonnée, ne sont plus à même de saisir l'hybridation grandissante des types de déplacement contemporains et les intentionnalités qui leur sont associées; plus que jamais les limites entre les mobilités touristiques, résidentielles, économiques, professionnelles, scolaires, médicales, affinitaires, deviennent floues et mouvantes, brouillant les catégorisations classiques des mobilités établies selon les motivations. Par ailleurs, non seulement ces mobilités s'hybrident de manière synchronique, mais elles s'inscrivent également de façon diachronique dans un continuum où elles alternent et s'enchaînent selon une logique dialectique et parfaitement réversible. 
Enfin, la plaisance au long cours illustre de manière assez éloquente les deux facettes d'un habiter singulier, où l'ancrage et la mobilité sont non seulement articulés, mais dans ce cas précis littéralement confondus.

Au fond, tout comme les backpackers, les big trippers ou les camping-caristes, les plaisanciers envisagent leur voyage comme un mode de vie, qui mêle intimement quotidien et hors-quotidien, ici et ailleurs, sédentarité et nomadisme, participant à «la recomposition en profondeur du 'rangement du monde' installé au XXe siècle entre les espaces, temps et pratiques de l'Ici (ville, quotidien, travail) et de l'Ailleurs (nature, hors quotidien, loisir)» (Bourdeau, 2012, p. 40).

Ces pratiques émergentes ne sont pas sans conséquences pour les territoires d'accueil en ce qu'elles modifient considérablement le rapport aux liens et aux lieux. La dimension éphémère et liquide de ces modes d'habiter poly-topique et "polychronique complexifie la gestion publique des territoires d'accueil et produit de la discontinuité, voire de la fragmentation sociale et spatiale entre les groupes sédentaires et ceux qui "passent ", entre ceux qui ont accès à la mobilité et ceux qui ne l'ont pas. Les fortes inégalités en termes de capitaux économiques, sociaux et mobilitaires entre les plaisanciers et les populations îliennes produisent de nouveaux rapports de domination sociale et politique qui questionnent les modalités d'un vivre ensemble insulaire, et invitent à une relecture nécessairement critique et postcoloniale des mobilités contemporaines.

\section{BIBLIOGRAPHIE}

ALLEMAND S. (2004), « La mobilité comme capital », Sciences Humaines, no.145, pp. 25.

BACQUE M.H., FOL S. (2013), « L'immobilité, facteur d'injustice spatiale ?», in Fol S., LehmanFrisch S., Morange M., Ségrégation et justice spatiale, Presses universitaires de Paris Nanterre, pp. 315-332.

BAUMAN Z. (2000), Liquid modernity, Cambridge, Polity Press, 240 p.

BAUMAN Z. (2013), La vie liquide, Hachette, Pluriel, 266 p.

BELTON-CHEVALLIER, L. (2015), « Prendre en compte l'immobilité dans les imaginaires de la mobilité ", Sociologies [Online], Files, La mobilité dans tous ses états. Représentations, imaginaires et pratiques. URL $:$ http://journals.openedition.org/sociologies/5155

BENSON M., O'REILLY K. (2009), Lifestyle Migration. Expectations, Aspirations and Experiences. London, Ashgate, $168 \mathrm{p}$.

BERROIR, S. et al. (2017), « Mobilité au quotidien et ancrage local dans les espaces périurbains », Annales de géographie, no713, p. 31-55.

BOLTANSKI L., CHIAPELLO E. (1999), Le nouvel esprit du capitalisme, Paris, Gallimard, coll. « NRF Essais », $980 \mathrm{p}$. 
BONERANDI E. (2004), « La mobilité des populations pauvres dans les espaces anciennement industrialisés. Pour une relecture de la pauvreté en milieu rural », Actes du colloque franco-espagnol de géographie rurale, Habiter et vivre dans les campagnes de faible densité, Foix, 15-16 septembre 2004.

BONNEMAISON, J., (1996). Les fondements géographiques d'une identité - l'Archipel du Vanuatu, Essai de géographie culturelle - Livre 1: Gens de pirogue et gens de la terre, Livre II : Les gens des lieux, Paris, Orstom, 460 p et 562 p.

BORJA S., COURTY G., RAMADIER T. (2013), « Mobilité : la dynamique d'une doxa néolibérale », Regards Sociologiques, $n^{\circ}$ 45-46, pp. 5-10. https://www.researchgate.net/publication/ 316439161_Mobilite_la_dynamique_d'une_doxa_neoliberal

BORJA S., COURTY G., RAMADIER T. (2014), « Trois mobilités en une seule ? », EspacesTemps.net [En ligne], Travaux, 2014 | Mis en ligne le 14 octobre 2014, https://www.espacestemps.net/articles/ trois-mobilites-en-une-seule/

BOURDEAU P. (2012), « Le tourisme réinventé par ses périphéries? », in Bourlon F., Osorio M., Mao P., Gale T., Explorando las nuevas fronteras del turismo. Perspectivas de la invetigacion en turisme, Nire Negro, pp. 31-48. http://hal.univ-grenoble-alpes.fr/hal-01002411/file/ Bourdeau_Tourisme_Invente.pdf

CAILLY L. (2014), « Les habitants du périurbain tourangeau à l'épreuve d'un changement de modèle : vers une recomposition des modes d'habiter? L'exemple des habitants de la ZAC des Terrasses de Bodets à Montlouis-sur-Loire ", Norois, no. 231, pp. 75-90. https:// journals.openedition.org/norois/5097

CATTAN, N. (2014), « Repenser la territorialité. L'apport du croisement mobilité et genre », BSGLg [En ligne], 62 (2014/1) - Questions et débats en géographie, URL : https://popups.uliege.be : 443/0770-7576/index.php ?id =173.

CERIANI-SEBREGONDI G. (2003), « Migrations internationales : vers un nouvel habiter ? ", Travaux de l'Institut Géographique de Reims, vol. 29-30, no.115-118, pp. 59-74. https://www.persee.fr/doc/ tigr_0048-7163_2003_num115_1463

CROUCHER, S. (2012). "Privileged Mobility in an Age of Globality," Societies, MDPI, Open Access Journal, vol. 2(1), pages 1-13.

DebarbieuX, B., Del BIAGGiO, C., PETITE, M., (2014), « Spatialités et territorialités du tourisme », Civilisations, 57 | 2008, 75-89.

DEBARBIEUX, B., (2014), « Enracinement - Ancrage - Amarrage : raviver les métaphores », L' Espace géographique, 2014/1, T.43, pp. 68 à 80.

DUNCAN T., COHEN SA., THULEMARK M. (2013), Lifestyle mobilities : Intersections of travel, leisure and migration, Ashgate, Farnham, $261 \mathrm{p}$.

FEILDEL B., MARTOUZET D. (2012), « La mobilité comme modalité de l'ancrage : enrichir l'évaluation de la durabilité des espaces périurbains ", Recherche Transports Sécurité, NecPlus, vol 28, no. 3-4, pp. 271-289. https://link.springer.com/article/10.1007/s13547-012-0042-8

FRETIGNY J.B. (2013), « La catégorie de mobilité naturalisée par les lieux de passage : étude de la publicité à l'aéroport de Roissy Charles-de-Gaulle », Regards Sociologiques, vol 1, no. 45-46, pp. 47-69.

GALLEZ C., KAUFMANN V. (2009), « Aux racines de la mobilité en sciences sociales : Contribution au cadre d'analyse socio-historique de la mobilité urbaine ", in Flonneau M., Guigueno V, De l'histoire des transports à l'histoire de la mobilité ?, Presses Universitaires de Rennes, pp. 41-55. https://halshs.archives-ouvertes.fr/halshs-00570341v2/document 
GAUGUE A. (2011), « Apprendre à naviguer autour du monde », in G. Brougère et G. Fabbiano (dir.), Tourisme et apprentissages, Actes du colloque de Villetaneuse (16-17 mai 2011), Villetaneuse, EXPERICE - Université Paris 13.

GAUGUE A. (2011), « Les lieux d'un tour du monde à la voile. Exploiter la diversité des lieux », in mondes du tourisme «Tourisme et mondialisation $", 9 \mathrm{p}$.

GAUGUE A. (2013), « Le tourisme peut-il être un mode de vie permanent ? L'exemple des plaisanciers au long cours », in H. François, P. Bourdeau, L. Perrin-Bensahel (dir.), Fin ( ?) et confins du tourisme. L'Harmattan, $220 \mathrm{p}$.

GAUGUE A. (2014), « La conquête plaisancière de la haute mer », Géoconfluences. http:// geoconfluences.ens-lyon.fr/informations-scientifiques/dossiers-thematiques/oceans-etmondialisation/corpus-documentaire/la-conquete-plaisanciere-de-la-haute-mer

GAUGUE A. (2018), «Les plaisanciers au long cours des voyageurs pas comme les autres » in revue Espaces, no $343,7 \mathrm{p}$

HANNAM K., SHELLER M., URRY J. (2006), « Mobilities, Immobilities and Moorings (Editorial) », Mobilities, vol. 1, no.1, pp. 1-22. https://edisciplinas.usp.br/pluginfile.php/363222/mod_resource/ content/0/7-Urry_Mobilities.pdf

HUI, P. et al., 2008, "Human mobility models and opportunistic communications system design", Phil. Trans. R. Soc, 366, 2005-2016.

JOSSO S. (2010), L'habitation du voilier de plaisance en croisière familiale, anthropologie d'un espace et des gouvernementalités en jeu, Thèse d'Anthropologie sociale et ethnologie. Université de Bretagne Occidentale (UBO), Brest, 1154 p. https://hal.laas.fr/tel-01996410/document

KAUFMANN, V. (2008), Les paradoxes de la mobilité, bouger, s'enraciner, Presses polytechniques et universitaires romandes, Lausanne, $115 \mathrm{p}$.

KESSELRING, S. (2006), "Pioneering Mobilities : New Patterns of Movement and Motility in a Mobile World". Environment and Planning A. 38. 269-279.

KNAFOU R. (1998), La planète « nomade ». Les mobilités géographiques d'aujourd'hui, Paris, Belin, $250 \mathrm{p}$.

LARSEN, J., KAY W. AXHAUSEN, KAY W. \& URRY, J. (2006), “Geographies of Social Networks : Meetings, Travel and Communications", Mobilities, $1: 2,261-283$

LASSEN, C. et al. (2006), "Virtual mobility and organizational reality - a note on the mobility needs in knowledge organisations", Transportation Research Part D : Transport and Environment, Volume 11, Issue 6, p. 459-463.

LAZZAROTTI, O. (2006), Habiter, la condition géographique, Paris, Belin, 297 p.

LAZZAROTTI, O, FRELAT-KAHN, B. (dir.) (2012), Habiter : vers un nouveau concept, Paris, Armand Colin, $256 \mathrm{p}$.

LAZARROTTI, O. (2014), Habiter le monde, Documentation photographique, $\mathrm{n}^{\circ} 8100$, Paris, La Documentation française, $64 \mathrm{p}$.

LE BIGOT B. (2017), Penser les rapports aux lieux dans les mobilités privilégiées, Étude croisée des backpackers en Thailande et des hivernants au Maroc, thèse de Géographie, Paris I, 556 p. http:// www.theses.fr/2017PA01H018

MARTIN, N., BOURDEAU, P., \& DALLER, J. F. (2012). Migrations d'agrément : du tourisme à l'habiter, Paris, Editions L'Harmattan, 412 p. 
MINCKE C., KAUFMANN V. (2017), « Mobilités changeantes, mobilités intriquées. », EspacesTemps.net [En ligne], Mis en ligne le 29 mars 2017. https://www.espacestemps.net/ articles/mobilites-changeantes-mobilites-intriquees/

MINCKE C., MONTULET B. (2019), La société sans répit. La mobilité comme injonction, Éditions de la Sorbonne, Collection Mobilités \& sociétés, 180 p.

MOITESSIER B. (1993), Tamata et l'Alliance. Mémoires, Arthaud, 401 p.

NESSI H. (2015), « Le rapport à l'espace pour dépasser l'opposition entre ancrage et mobilité », Les Cahiers Scientifiques du Transport, no.67 pp. 3-32. http://afitl.ish-lyon.cnrs.fr/tl_files/documents/ CST/N67/Nessi67.pdf

PARRAIN C. (2010), Territorialisation des espaces océaniques hauturiers. L'apport de la navigation à voile dans l'Océan Atlantique. Thèse de Géographie. Université de La Rochelle. https://tel.archivesouvertes.fr/tel-00713524

SEGALEN V. (2001), Les Immémoriaux, Paris, Classique, p. 319

SHELLER M., URRY J. (2006), « The new mobilities paradigm », Environment and Planning A, vol 38, pp. 207-226

STASZAK J-F. (2003), Géographies de Gauguin, Bréal, 255 p.

STOCK M. (2015), « habiter comme 'faire avec l'espace'. Réflexions à partir des théories de la pratique ", Annales de géographie, vol 4, no. 704, pp. 424-441 https://www.cairn.info/revueannales-de-geographie-2015-4-page-424.htm

STOCK M. (2018), « Habiter la ville en mouvements », in Asholt W., Calle-Gruber M., Heurgon E., Oster-Stierle P, L'Europe en mouvement - 1. à la croisée des cultures, Hermann, pp. 145-154.

URRY J. (2000), Sociology beyond Societies Mobilities for the Twenty-First Century, New York : Routledge.

URRY J. (2007), Mobilities. Cambridge, Polity, 335 p.

URRY J. (2012), « Qu'est-ce que le « tournant de la mobilité » ? », Forum Vies Mobiles - Préparer la transition mobilitaire. http://fr.forumviesmobiles.org/video/2012/12/10/quest-ce-quetournant-mobilite-453

VACHER L. (2004), « Le tourisme au Kimberley et le "Big trip" des retraités australiens », Le Globe, Revue genevoise de géographie, tome 144, pp. 41-55. https://www.persee.fr/doc/ globe_0398-3412_2004_num_144_1_1485

VINCENT-GESLIN, S., KAUFMANN V. (dir.), 2012, Mobilité sans racines. Plus loin, plus vite... Plus mobiles?, Paris, Descartes et Cie, Cultures mobiles, $142 \mathrm{p}$.

WEBER S. (2009), «Les mobilités induites par les migrations : émergence d'un champ circulatoire transnational », Méditerranée [En ligne], 113 | 2009, mis en ligne le 31 décembre 2011, http:// journals.openedition.org/mediterranee/3790

ZENEIDI D. (2002), Les SDF et la ville. Géographie du savoir survivre, Paris, Ed Bréal, 288 p.

\section{NOTES}

1. Tous les prénoms ont été changés pour garantir l'anonymat des enquêtés. 
2. Terme qui désigne la migration annuelle hivernale des retraités originaires principalement d'Europe de l'Ouest, des Etats-Unis ou du Canada vers des destinations au climat plus clément et au coût de la vie plus intéressant.

3. Uturoa est la commune principale de l'île de Raiatea.

4. La caisse de bord est un « pot commun » qui permet de payer toutes les dépenses communes de la vie sur un bateau.

5. Cf. http://www.levoyagedesuricat.com/journal-de-bord/marquises-2005-907-the-qwaoooowqeffect.html

6. Dans Les Immémoriaux, Segalen (un écrivain voyageur du tournant du XIXe-XXe siècle) associe l'île et la pirogue qu'il féminise toutes deux: "Cette pirogue était lourde et chevelue. Les hommes de Matavaï pensèrent à l'arrivée d'une île voyageuse » (p.60).

7. Extrait d'un entretien réalisé dans le cadre d'une autre enquête auprès de Polynésiens de Raiatea.

\section{RÉSUMÉS}

Après avoir traversé l'océan Atlantique, sillonné les îles Caraïbes et navigué à travers l'océan Pacifique, de nombreux plaisanciers font escale sur l'île de Raiatea en Polynésie française. Ces plaisanciers s'inscrivent dans des circulations qui peuvent se déployer sur plusieurs années et à différentes échelles. A partir d'un travail de terrain réalisé auprès de 13 plaisanciers installés à Raiatea, cet article souhaite identifier comment les mobilités des plaisanciers se structurent et construisent des espaces de vie. L'étude que nous présentons interroge de nouvelles formes de mobilités qui remettent en question les catégories classiques d'analyse. Elle propose une réflexion dialectique à partir de l'exemple emblématique d'un habiter où ancrage et mobilité se confondent en un seul lieu : le voilier.

After crossing the Atlantic Ocean, circumnavigating the Caribbean Islands and sailing through the Pacific Ocean many boaters stop on Raiatea Island in French Polynesia. Their journeys can extend over several spatial and temporal scales. Based on 13 boaters's interviews conducted in Raiatea, the article identifies how their mobilities are structured and the role they play in their life. This study deals with new kinds of mobilities that question the conventional categories often used to analyse mobility and provides a reflection on the relationship between mobilities and moorings, giving an iconic example where mooring and mobility merge together in one place : the sailboat.

\section{INDEX}

Thèmes : Carnets de recherches

Keywords : Mobility, mooring, boaters, Raiatea (French Polynesia)

Mots-clés : Mobilité, ancrage, plaisanciers, Raiatea (Polynésie-française) 


\section{AUTEURS}

\section{NATHALIE BERNARDIE TAHIR}

Professeure de Géographie, Université de Limoges. Laboratoire Geolab (Laboratoire de Géographie Physique et Environnementale). nathalie.bernardie-tahir[at]unilim.fr

\section{SARAH BERNARD}

Doctorante en Géographie, Université de Limoges. Laboratoire Geolab (Laboratoire de Géographie Physique et Environnementale). sarah.bernard[at]unilim.fr 